\title{
La motivación y el aprendizaje
}

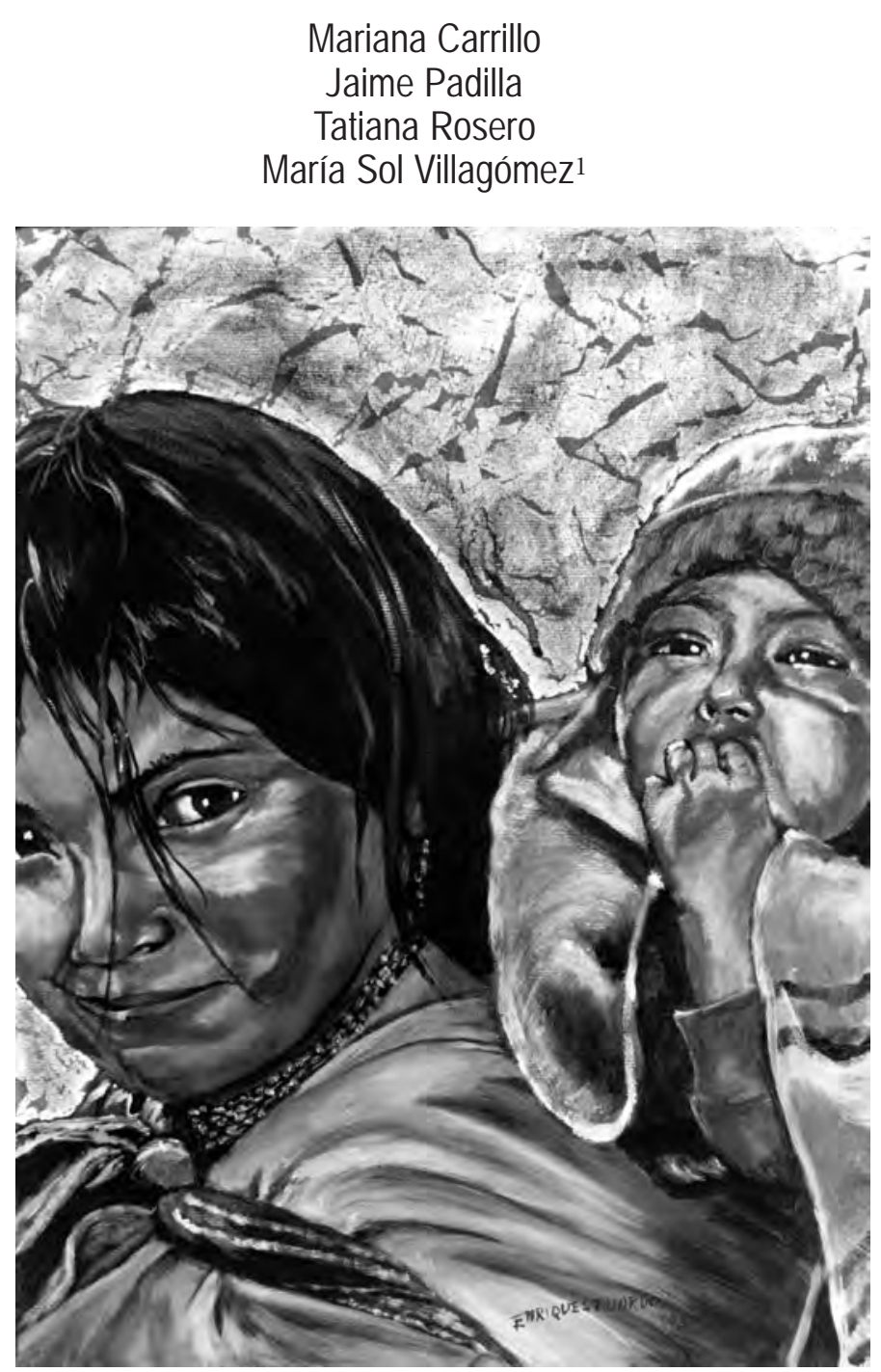

Niña con bebé. Lámina de oro y acrílico sobre tela. 2003

1 Mariana Carrillo: Especialista en Educación a Distancia. Directora de la Carrera de Pedagogía en la UPS - sede Cuenca; Jaime Padilla: Magíster en Gerencia y Liderazgo Educacional. Director del área de Educación; Tatiana Rosero: Máster en Docencia con mención en Educomunicación. Directora del Diploma en Docencia Universitaria en la UPS; María Sol Villagómez: Máster en Docencia con mención en Educomunicación. Directora de la Carrera de Pedagogía de la UPS - sede Quito. 
Si bien es cierto, que los resultados del aprendizaje dependen en gran medida de los conocimientos, habilidades y valores adquiridos o desarrollados previamente por los educandos; es indudable que también obedecen a la calidad del docente; es decir que, tanto los aprendizajes previos, cuanto la calidad del profesor constituyen los fundamentos del aprendizaje. Por otra parte, psicólogos y educadores, sociólogos y pedagogos, entre otros especialistas relacionados directamente con el quehacer educativo coinciden en señalar que el desempeño escolar pende, en gran medida, del grado o nivel de motivación que posea el estudiante.

Este artículo busca analizar los conceptos de motivación y aprendizaje, relacionar las implicaciones que existen entre ambos, y concluye con referencias para las aplicaciones didácticas en el área de educación.

\section{Concepto de motivación}

La motivación, del latín motivus (relativo al movimiento), es aquello que mueve o tiene eficacia o virtud para mover; en este sentido, es el motor de la conducta humana. El interés por una actividad es "despertado" por una necesidad, la misma que es un mecanismo que incita a la persona a la acción, y que puede ser de origen fisiológico o psicológico. Cada vez que aparece una necesidad, ésta rompe el estado de equilibrio del

Figura 1. Ciclo motivacional

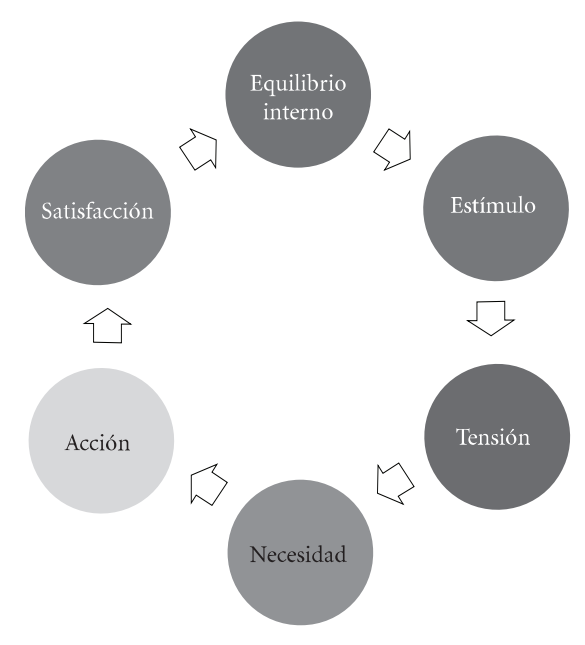

organismo y produce un estado de tensión, insatisfacción e inconformismo que lleva al individuo a desarrollar un comportamiento o acción capaz de descargar la tensión. Una vez satisfecha la necesidad, el organismo retorna a su estado de equilibrio anterior. La figura 1. muestra el esquema del ciclo motivacional.

Por tanto, ante una situación dada, la motivación determina el nivel con qué energía y en qué dirección actuamos. Una de las teorías más conocidas sobre la motivación es la que se grafica en la Jerarquía de Necesidades Humanas de Maslow (1956). Ésta coloca a las necesidades básicas o simples en la base de la pirámide y las relevantes o fundamentales en la cima; en este sentido, los cuatro primeros niveles son considerados como "necesidades de supervivencia"; al nivel superior lo denominó "motivación de crecimiento", o "necesidad de ser". A medida que la persona logra controlar sus necesidades de jerarquía inferior aparecen gradualmente necesidades de orden superior.

Si bien Maslow presenta este orden de prioridad, considerado como el orden normal o lógico, en diferentes etapas de nuestra vida y ante determinadas circunstancias, el orden puede variar; es más, existen casos de personas que anteponen a las necesidades fisiológicas, necesidades de autorrealización. 
Figura 2. Jerarquía de necesidades humanas de Maslow

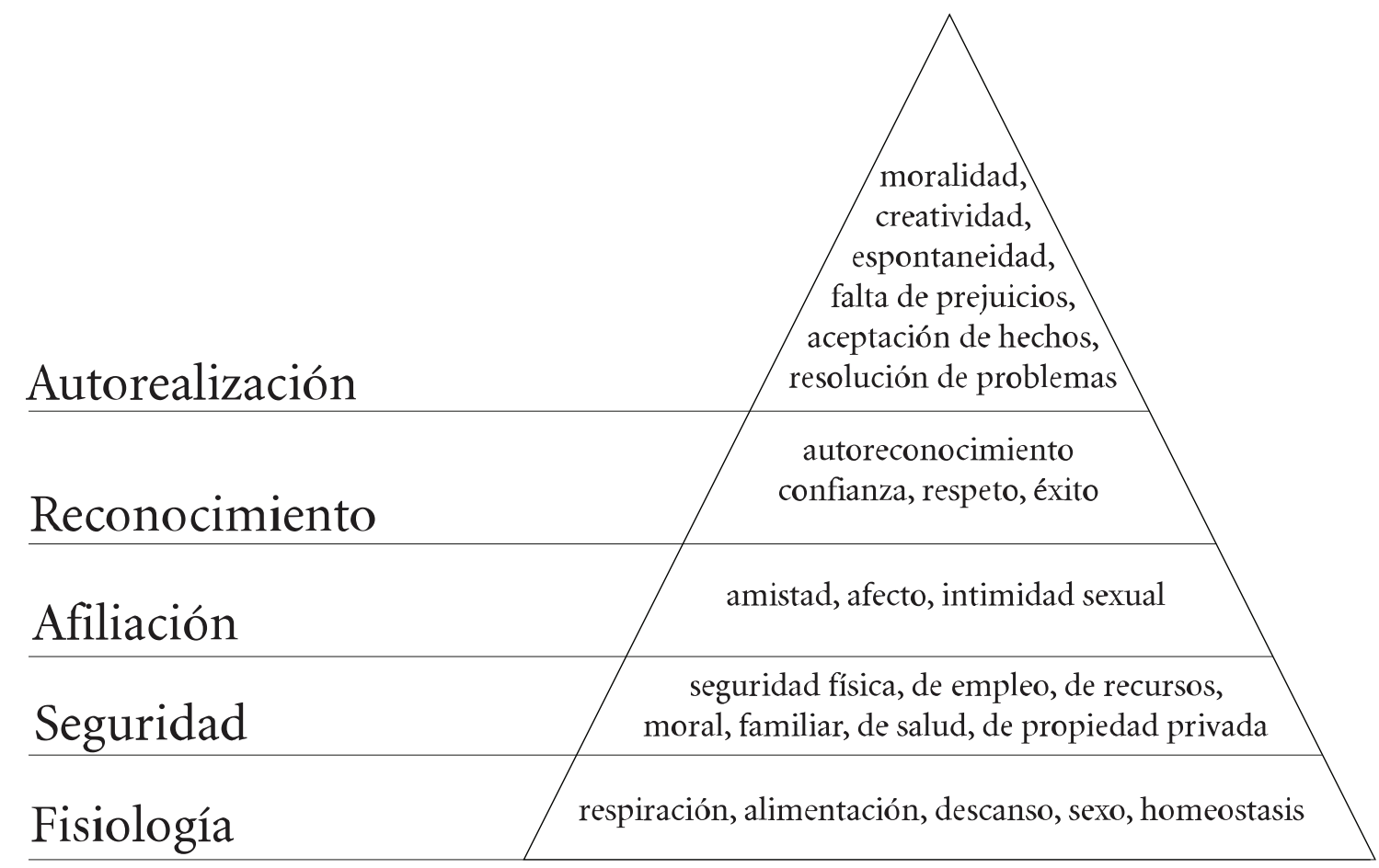

Aunque la mayoría de los especialistas coinciden en definir la motivación como un "conjunto de procesos implicados en la activación, dirección y persistencia de la conducta" (Beltrán, 1993; Bueno, 1995; McClelland, 1989, ...), el marco explicativo de cómo se produce la motivación, cuáles son las variables determinantes, cómo se puede mejorar desde la práctica docente, etcétera, son cuestiones no resueltas, y en parte las respuestas dependerán del enfoque psicológico que se adopte. En el cuadro 1 se realiza la comparación entre las teorías de Maslow, Herzberg y Alderfer lo que nos da una idea de esta unidad y diversidad.

Para sintetizar los tipos de motivaciones, es necesario considerar las siguientes dimensiones: primero diferenciar entre las internas y las externas; segundo diferenciar entre las positivas y las negativas. Al combinar esas dos dimensiones obtenemos los cuatro tipos de motivaciones (ver cuadro 2).

Tanto las motivaciones negativas que tratan de evitar un castigo, perjuicio o daño; cuanto las motivaciones estimuladas desde el exterior que obedecen a recompensas, no persisten. Únicamente la motivación intrínseca, que obedece a motivos internos es sostenible.

De la reflexión hasta ahora realizada podemos inferir que las personas actúan porque tienen ideas, deseos o metas que las impulsan y que la motivación depende de la voluntad; sin embargo, con el tiempo, las tendencias de la psicología disminuyeron el rol de la voluntad en la 
Educación y aprendizaje

Cuadro 1. Comparación entre las teorías de Maslow, Herzberg y Alderfer

\begin{tabular}{|c|}
$\begin{array}{c}\text { Teoría de la Jerarquía } \\
\text { de necesidades según } \\
\text { A. Maslow }\end{array}$ \\
\hline $\begin{array}{c}\text { Autorrealización } \\
\text { y satisfacción }\end{array}$ \\
\hline Estima y posición \\
o status \\
\hline Necesidades \\
Sociales \\
y de pertenencia \\
\hline \\
\hline Seguridad de \\
todos los tipos \\
\hline
\end{tabular}

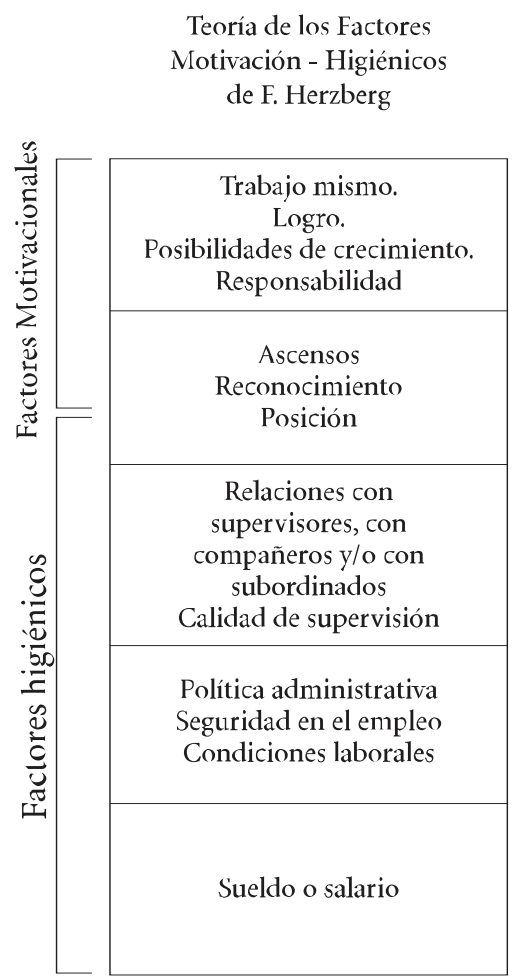

Teoría E-R-G

de Necesidades según

C. Alderfer

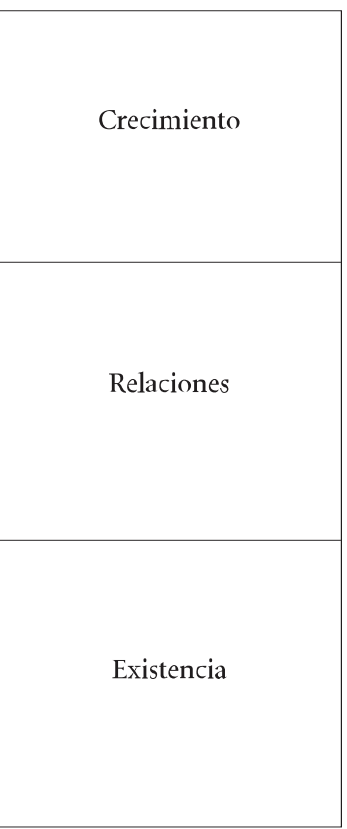

motivación, ya que, con frecuencia, la gente no hace las cosas porque quiere hacerlas solamente, sino porque tiene que hacerlas.

Actualmente existen dos grandes corrientes psicológicas o teorías con enfoques diferentes, por una parte los psicólogos conductistas, que sostienen que no están interesados en lo que ocurre dentro del sujeto, y consideran que el estímulo inicial está asociado a un refuerzo positivo que surge en el ambiente como consecuencia de una conducta. Por otra parte, los psicólogos cognitivos consideran e identifican una serie de procesos entre el estímulo y la respuesta tales como pensamientos, atribuciones, expectativas, etcétera. En tanto, en el enfoque conductista, el estímulo provoca directamente la respuesta, en el enfoque cognitivo el
Cuadro 2. Tipos de motivación

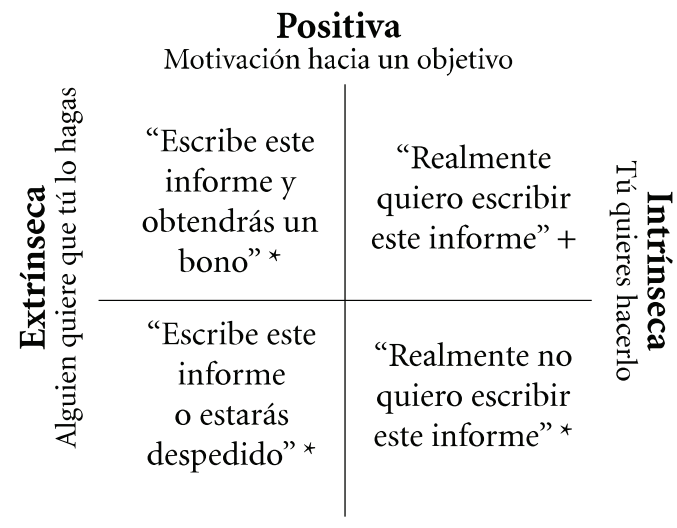

Negativa

Motivación por algo a evitar 
estímulo activa una variedad de sucesos internos que provocan un comportamiento. Y estos sucesos internos son los que interesa conocer, promover y modelar porque de ellos depende la respuesta (Gagné, 1985).

Brunner (1966) identificó tres formas de motivación intrínseca:

- De curiosidad, que satisface el deseo de novedad. Surge así el interés por los juegos y las actividades constructivas y de exploración.

- De competencia, se refiere a la necesidad de controlar el ambiente. Si eso sucede, aparecerá el interés por el trabajo y el rendimiento.

- De reciprocidad: alude a la necesidad de comportarse de acuerdo con las demandas de la situación. ción:

Hay cuatro fuentes principales de motiva-

- Nosotros mismos (equilibrio emocional, pensamiento positivo, aplicación de buenas estrategias, seguimiento de rutinas razonables, etcétera).

- Los amigos, la familia y los colegas, en realidad, nuestros soportes más relevantes.

- Un mentor emocional (real o ficticio).

- El propio entorno (aire, luz, sonido, objetos motivacionales).

Las diversas escuelas de la psicología se han ocupado del estudio de la motivación como uno de los principales procesos básicos de la conducta con indudables componentes fisiológicos subyacentes. Los estudios de la motivación han sido también muy relevantes en el campo de la psicología aplicada dentro de dominios tan diversos como el laboral y la selección de personal, la psicología clínica, el psicoanálisis, la publicidad, la Internet, etcétera. A su vez, existen diversas recomendaciones para sentirse motivados.

\section{Mecanismos que regulan el aprendizaje}

Al preguntarnos cómo aprendemos, recurrimos a la necesidad de encontrar una explicación que nos permita comprender cómo suceden los aprendizajes.

Para Ausubel, uno de los principales exponentes de la pedagogía constructivista, los aprendizajes significativos son aquellos que se integran a la estructura cognitiva del sujeto que aprende; para que esto suceda se determinan unos mínimos requeridos tanto en el objeto a aprender como en el sujeto que aprende. Por su parte el objeto de aprendizaje debe ser funcional, integrable, potencialmente significativo e internamente coherente, mientras que el sujeto que aprende debe disponer de las estructuras cognitivas necesarias que le permitan establecer relaciones con el nuevo conocimiento, es decir conocimientos previos y presentar una actitud favorable frente al nuevo aprendizaje.

La motivación es aquella actitud interna y positiva frente al nuevo aprendizaje, es lo que mueve al sujeto a aprender, es por tanto un proceso endógeno. Es indudable que en este proceso en que el cerebro humano adquiere nuevos aprendizajes, la motivación juega un papel fundamental. Pero, además de una actitud favorable para aprender, el sujeto que aprende debe disponer, según los estudios de Ausubel, de las estructuras cognitivas necesarias para relacionar los conocimientos previos con los nuevos aprendizajes. El cerebro humano se encuentra programado para la supervivencia, por lo que está potencialmente preparado para aprender, este aprendizaje implica un proceso dual, por un lado necesita y registra lo familiar y, por el otro busca lo novedoso para aprender. Mediante el aprendizaje se da sentido a aquello que es nuevo en relación con lo ya conocido, en este proceso, los estímulos del medio juegan un papel fundamental. 
Según Ian Gilbert (2005) “El cerebro está diseñado para: la supervivencia... Cuando nos enfrentamos a una situación de aprendizaje, hay una parte de nuestro cerebro que se pregunta: ¿Necesito este aprendizaje para sobrevivir? ¿Sí o no? En caso afirmativo podemos seguir con el aprendizaje. Sin embargo, si la respuesta es negativa, olvidémonos de todo". En este sentido, la motivación para el nuevo aprendizaje vuelve a considerarse como un factor determinante del mismo.

Las investigaciones de autores como Levy y Austin acerca del funcionamiento de los hemisferios cerebrales afirman que éstos tienen diferentes estilos para procesar información, el hemisferio izquierdo es conceptualizante, secuencial, lógico, lineal de las partes al todo, temporal y analítico y el hemisferio derecho, se relaciona con las emociones, el arte, la creatividad, la imaginación y la información no verbal, es simultáneo, espacial, asociativo, del todo a las partes, es atemporal. La vinculación de las funciones y la transmisión de información de un hemisferio a otro se dan a partir del cuerpo calloso o comisuras cerebrales que actúan como integradoras.

El cerebro humano tiene dos hemisferios que trabajan interconectadamente, el lado derecho es creativo y el derecho es académico o detallista. "Las habilidades mentales de los hemisferios, que fueron identificadas por el premio Nobel Roger Sperry en 1960, están distribuidas e integradas en toda la corteza cerebral. Por ejemplo, si escuchamos una canción, las palabras se procesarán en el hemisferio izquierdo mientras que la música se procesará por el derecho" (Ortiz, 2001).

De los estudios acerca de los sistemas cerebrales se desprenden las investigaciones de las diferentes inteligencias humanas como la teoría de las inteligencias múltiples de Gardner, que nos lleva a la comprensión de las potencialidades que tiene cada sujeto para aprender en las diversas áreas del saber. Estos mecanismos que regulan los aprendizajes vendrían mediados por procesos y capacidades individuales. La pirámide del aprendizaje (figura 3) es un instrumento que puede ayudar a considerar las distintas formas de aprender de los estudiantes orientando nuestras prácticas pedagógicas hacia su aprendizaje significativo.

Martiniano Román y Eloísa Diez (2005), señalan algunos mecanismos que regulan el proceso del aprendizaje:

1. Evitar las críticas negativas ante los intentos de colaboración de los alumnos.

2. Estructurar la docencia en el aula de forma no excesivamente autoritaria mezclando la directividad con la aceptación de las decisiones de los alumnos.

3. Programar trabajos en grupo o sesiones donde cada alumno pueda colaborar según su nivel.

4. Valorar positivamente los comportamientos de trabajo o de estudio o en su defecto las aproximaciones.

Figura 3. Pirámide del aprendizaje

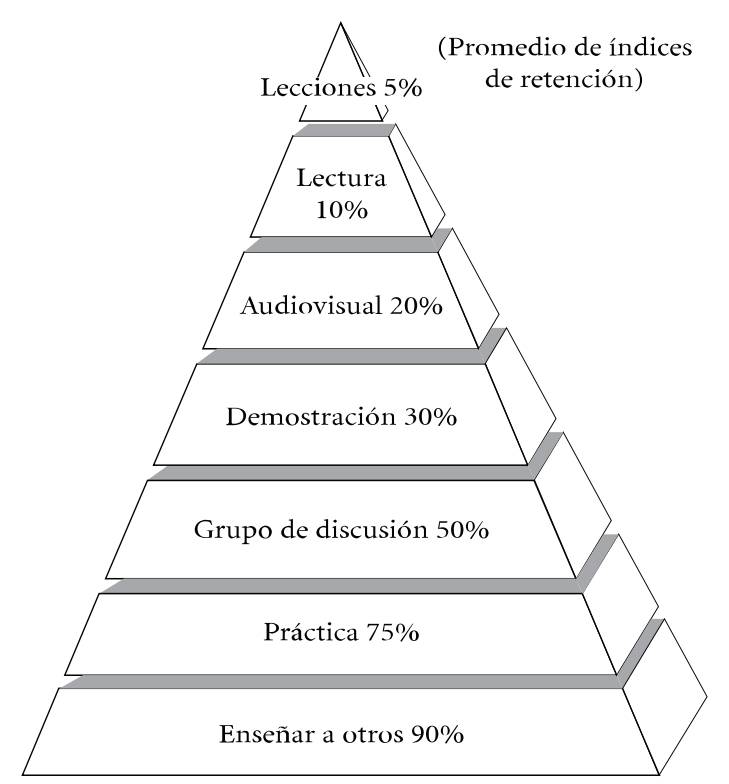


5. El reconocimiento del éxito de un alumno o de un grupo de alumnos motiva más que el reconocimiento del fracaso y si aquel es público mejor.

6. Conocer las causas del éxito o del fracaso en una tarea determinada aumenta la motivación intrínseca.

7. El aprendizaje significativo crea motivación, no ocurre lo mismo con el aprendizaje memorístico y repetitivo.

8. Programar los contenidos y enseñarlos de manera que los alumnos puedan comprenderlos y aplicarlos con un nivel medio de dificultad.

9. Cuidar de que los alumnos con un bajo nivel de motivación consigan pequeños éxitos académicos para que aspiren en un futuro próximo hacia metas que exigen esfuerzos superiores.

10. Tener presente que los alumnos con baja motivación, en un principio suelen manifestar cierta resistencia a abandonar su deficiente situación motivacional puesto que temen que el posible cambio pueda aumentar su, ya de por sí, precaria situación.

11. Fomentar el trabajo cooperativo frente al competitivo.

12. Presentar tareas asequibles a las posibilidades de los alumnos.

13. Programar las actividades de la clase para que los alumnos puedan frecuentemente tomar decisiones, el profesor que da autonomía en el trabajo promueve la motivación de logro y la autoestima, aumentando así la motivación intrínseca.

14. Promover actividades en las que los riesgos de fracaso son moderados.

15. Llevar la clase con un nivel medio de ansiedad y evitar las situaciones extremas de máxima ansiedad o de aburrimiento.

16. Programar sesiones de diálogo por grupos de manera que los alumnos menos motivados puedan expresar sus opiniones sin miedo a ser rechazados por sus compañeros.

17. Realizar actividades o trabajos fáciles para los alumnos poco motivados, de manera que pueda valorar sus éxitos y su relativa dedicación.

18. Las tareas creativas son más motivantes que las repetitivas.

\section{Acciones didácticas que favorecen la motivación}

Hay una historia ya clásica en la que se narra cómo un ser humano abandonaba la tierra y viajaba, durante un tiempo, a la velocidad de la luz. A su regreso al mundo, él apenas tenía unos años más pero para el resto había transcurrido muchísimo tiempo. Nada menos que varios siglos. De su desconcierto - no reconocía en nada algo conocido. Sólo le consoló el único sitio que seguía igual que siempre: la escuela.

Bazarra, 2005.

Todo proceso educativo se articula en el juego de sus elementos didácticos, éstos forman un sistema coherente que interactúa y favorece el logro de los objetivos de aprendizaje.

Desde este planteamiento básico el itinerario a seguir en los procesos educativos se apega a principios básicos (Nérici, 2005: 158-162) de la Didáctica General que se aplica a todas las disciplinas y áreas del conocimiento:

- Partir de lo próximo a lo remoto (asociar hechos o elementos de referencia próximos en el tiempo/espacio para facilitar la asociación con otros de la misma naturaleza).

- Partir de lo concreto para llegar a lo abstracto (partir de conocimientos/experiencias previas, ejemplificaciones, esquemas, vivencias...poniendo en diálogo al estudiante con la realidad).

- Partir de lo conocido para llegar a lo desconocido (articular lo nuevo con lo conocido estableciendo una relación lógica, psicológica o analógica de modo que adquiera significación para el estudiante).

- Individualización. 


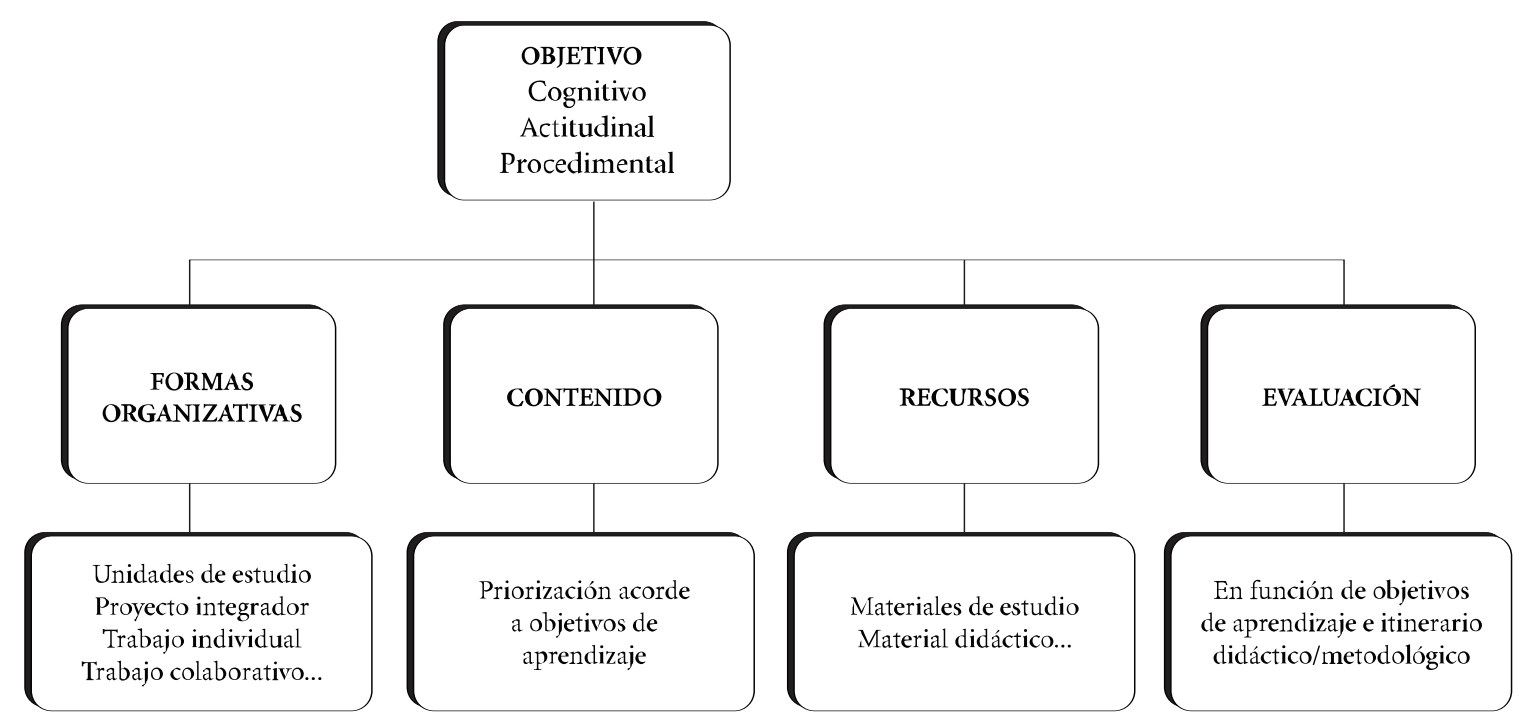

- Libertad.

- Autoactividad.

- Autodisciplina.

- Autonomía.

- Actividad.

- Participación.

- Respeto a la personalidad del estudiante.

- Principio de realidad.

- Principio de adecuación.

- Adecuación al nivel de enseñanza.

- Adecuación al nivel de desarrollo del educando.

- Adecuación a la comunidad socioeconómica y cultural de la comunidad.

- Principio de ordenamiento.

- Propender a la transferencia.

- Clarificar los objetivos previstos.

- Provocar la reacción del educando.

- Aspirar a la perfección (sin exigir perfección, sino motivar, acompañar y retroalimentar las producciones para el logro de mejores de- sempeños).

- Corregir los errores.

- Integrar e irradiar.

Parafraseando a Morin es pertinente señalar que el cambio en la escuela iniciaría con un "cambio de mentalidad" que nos permita mirar, leer, sentir, interpretar la realidad con ojos nuevos. Para mirar de modo diferente es preciso tomar distancia de la cotidianidad y ser autocríticos de nuestras prácticas regulares en el aula y así repensar nuestros roles y acciones metodológicas y didácticas.

Veamos algunas posibilidades para hacer prácticas exitosas y renovadas en el aula:

1. Ubiquemos los deberes por encima de los derechos personales y grupales, reflexionando si no caemos en el hecho de que "frente a todo lo que reclamamos, estamos aún dispuestos a dar muy poco" (Nérici, 2005: 40).

2. Asumamos que todo aprendizaje es un interaprendizaje que se construye de modo 
cooperativo entre docentes y estudiantes y estudiantes entre sí.

3. Conozcamos la materia y estemos al día con el desarrollo de sus propias disciplinas, de modo que puedan simplificar $y$ clarificar conceptos básicos y complejos para que el estudiante llegue motivado a la esencia de los contenidos.

4. Valoremos el conflicto como fuente de conocimiento y aprendizaje, pues desarrolla la creatividad, la observación, la reflexión y la escucha.

5. Apliquemos la pedagogía de la pregunta.

6. Prefiramos trabajar en las asignaturas pocos conceptos, pero con un avance en profundidad con una real discusión de éstos.

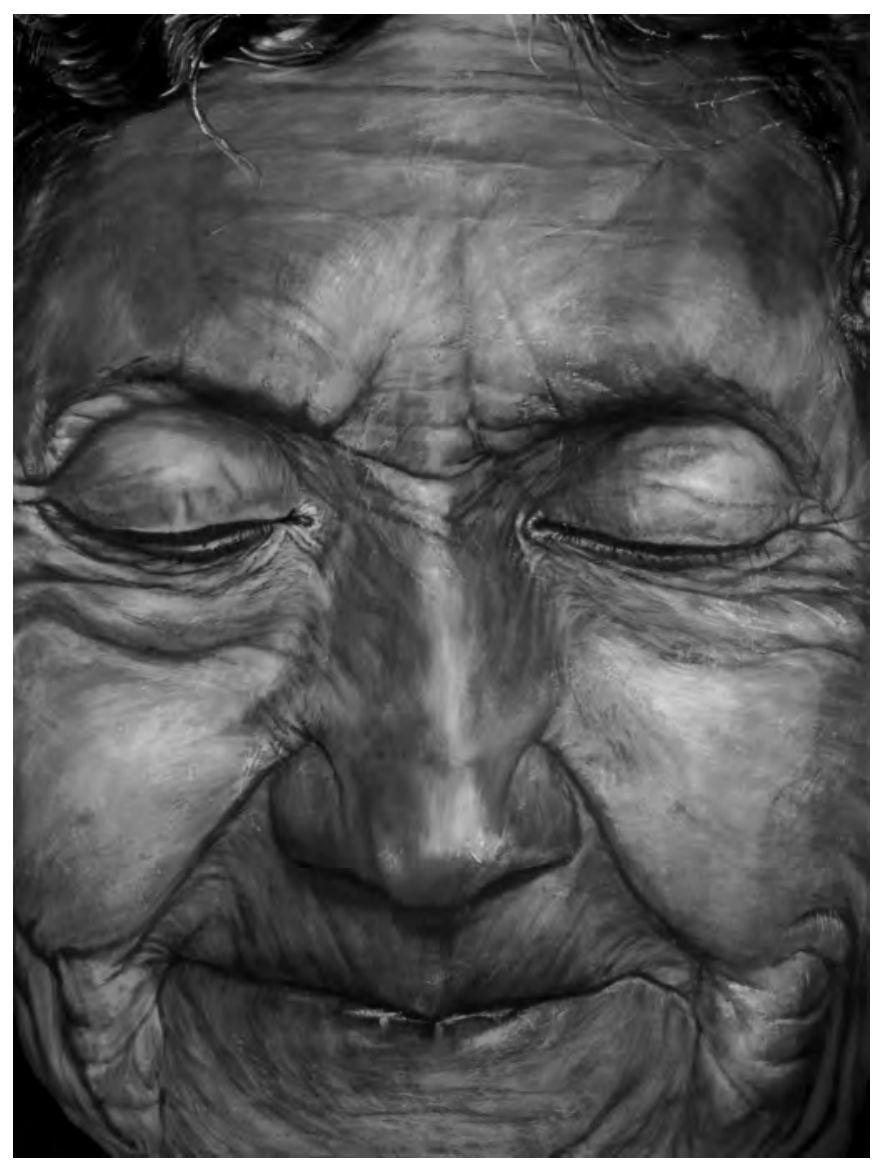

Quilago (32 módulos). Acrílico sobre tela. 2004
7. Reflexionemos y valoremos la utilidad de los conceptos, métodos y técnicas que se trabajan en las asignaturas de modo que permitan una sistematización del conocimiento y una reflexión sobre la forma en que fueron enseñados y aprendidos.

8. Construyamos ambientes físicos y emocionales propicios para el aprendizaje. Estas consideraciones pueden motivar una mejor participación, generar familiaridad y bienestar.

9. Creamos en la capacidad e interés por aprender que tienen sus estudiantes.

10. Valoremos la profesión docente reconociendo que la enseñanza es un acto de fe (que permite creer en el otro) y de libertad, que también requiere de motivación, estímulo y reconocimiento.

Para Gardner y cols. (2000) hasta los profesionales avezados pueden tener dificultades para trabajar bien si no se les ofrece alguna forma de reconocimiento o evidencia de aprecio, también necesitan motivación. La pericia es un proceso que consume y exige tiempo. Si no se obtiene una satisfacción personal que no esté intimamente ligada a alguna forma de reconocimiento público, es difícil que se quiera perseverar.

Miguez, 2006.

Entonces, ¿esperamos hasta que nos lleguen los estudiantes ideales y perfectamente motivados o les pedimos que esperen al o la docente ideal con quien siempre han soñado o nos empeñamos en formar adultos de calidad siendo nosotros mismos individuos con calidad humana y profesional?

\section{Motivaciones que generan aprendizajes}

1. Interés por el tema de trabajo. Este aspecto es básico y obvio, el interés que tenga el alumno por el tema concreto de estudio interviene en su motivación para el aprendizaje, un tema interesante desencadena con facilidad 
el esfuerzo necesario para aprenderlo.

2. El aprendizaje cooperativo. Es el aprendizaje que permite la interdependencia entre sus miembros, se organizan en pequeños grupos heterogéneos que trabajan en forma coordinada para alcanzar sus metas y resolver sus tareas académicas es una motivación donde se establecen las relaciones intersubjetivas, responsabiliza y se compromete con su propio aprendizaje y el de sus compañeros, su éxito es el de todos, por lo que sus objetivos son comunes a todo el grupo. Piaget sustenta que el Aprendizaje Cooperativo, es uno de los cuatro factores que intervienen en la modificación de estructuras cognitivas: la maduración, la experiencia, el equilibrio y la transmisión social.

3. Sentimiento de competencia. Es un factor clave en la motivación de muchos alumnos/as por el estudio, el sentirse competente le supone al discente pensar que puede aprender, lo que favorece que tenga sentido realizar el esfuerzo necesario para conseguirlo. Debemos enseñar a los alumnos a ser conscientes de este aspecto de su motivación y del papel que ellos tienen para mejorar progresivamente su capacidad para aprender.

4. Proyecto personal. Este motivo es el más general y en muchas ocasiones el más difícil, tener un proyecto supone ver el trabajo escolar como un medio para irlo consiguiendo; la sensación de estar haciendo lo que se quiere y conviene hacer, es un elemento muy significativo de madurez que favorece la disposición a esforzarse en las tareas escolares, hay una estrecha vinculación entre los aprendizajes con acento constructivista y el fomento de valores y proyectos.

5. Sentir ayuda del profesor. Isabel Solé sostiene que: la motivación no es algo que viene dado, sino que se construye en las propias situaciones de enseñanza y aprendizaje, la relación entre los alumnos y el profesor siempre es interactiva, su influencia es mu-

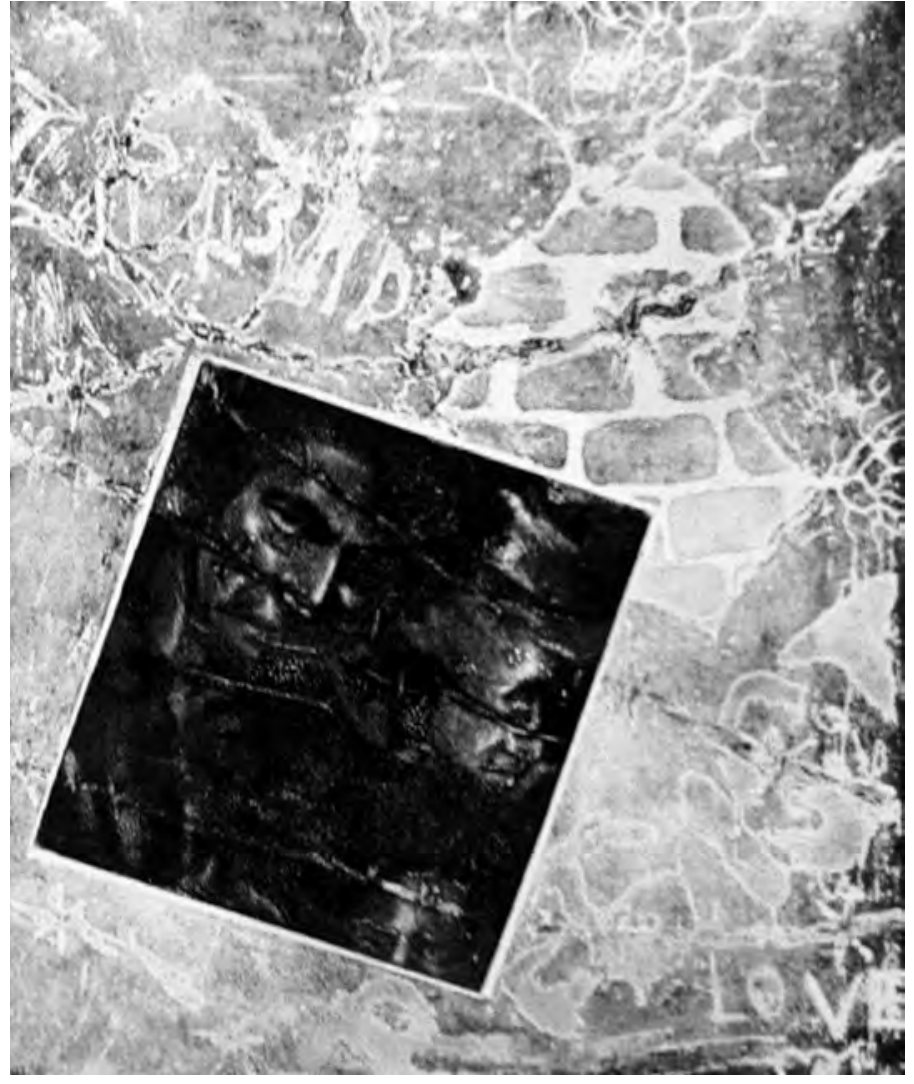

El triunfo de la libertad es el nombre de una calle I. Detalle. Roll up. 1996

tua; se trata de un compromiso humano, singular, con cada alumno, para ellos tiene más sentido llevar a cabo su actividad intelectual si perciben que el profesor quiere ayudarles de ésta manera entendido este aspecto, incluye todos los motivos que tratamos: interesarle sobre el tema, procurarle éxito, promover proyectos, organizar trabajos cooperativos, etcétera.

6. Sentir ayuda de los compañeros. Los compañeros suponen una fuente de información y modelo de proyecto de futuro, en parte, la motivación está determinada por los sentimientos que se producen al colaborar, ayudar o ser ayudado por los compañeros, el profesor no puede ser el único que enseña, los alumnos son también fuente de información 
y ayuda; los proyectos de trabajo en equipo y la enseñanza tutorizada entre alumnos promueven la responsabilidad y animan el esfuerzo. Los beneficios de las relaciones entre alumnos no ocurren de forma automática, requieren de la intervención del profesor y de un trabajo sostenido en el tiempo.

\section{Bibliografía}

- AZNAR, Pilar (coordinadora): Teoría de la educación. Un enfoque constructivista. Valencia: Editora Tirant Lo Blanch. 1999

- BAIN, Kein: Lo que hacen los mejores profesores de universidad, Publicaciones de la Universidad de

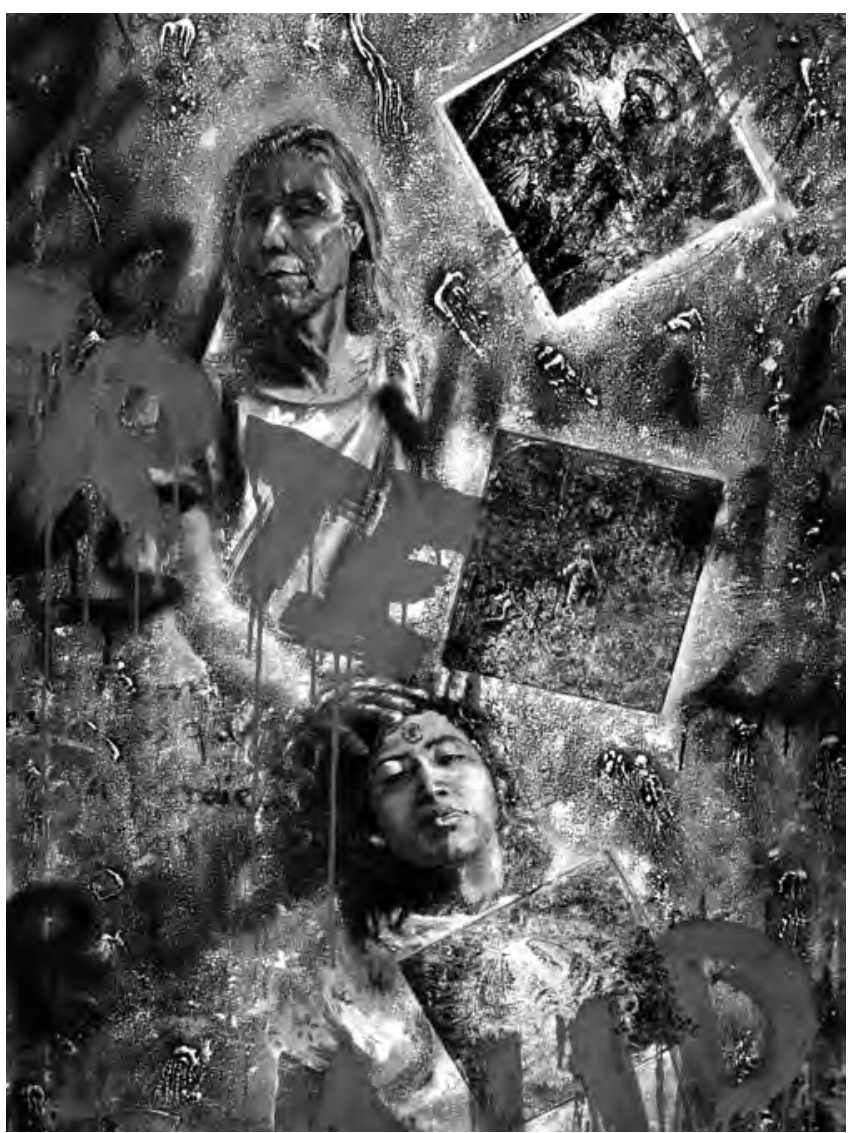

Puntos de convergencia. Acrílico sobre tela. 1996
Valencia. Barcelona. 2005

- BAZARRA, Lourdes y otros: Ser profesor y dirigir profesores en tiempos de cambio. Madrid: Narcea Ediciones. 2005

- BELTRÁN, Jesús: "Procesos, Estrategias y Técnicas de Aprendizaje”. Síntesis por la Gestión Escolar de Fundación Chile.

- GILBERT, Ian: Motivar para aprender en el aula, Paidós educadores.

- MONEREO, C. M. Castelló, Clariana y otros: Estrategias de enseñanza y aprendizaje. Formación del profesorado y aplicación en el aula, Barcelona: Graó. 1994

- NÉRECI, Imídeo: Hacia una didáctica general dinámica, Argentina: Kapelusz. 1973.

- ORTIZ, Elena María: El Cerebro en la educación de la persona. Editorial Bonum. 2001

- PÉREZ, Antonio: La motivación. Granada. 2009

\section{Bibliografía de Internet}

- AutorStream, web disponible en http://www.authorstream.com/Presentation/campanita-28111inteligencias-ltiples-multiples-education-ppt-powerpoint/

- Blog Recursos Educativos, disponible en http://anita-recursoseducativos.blogspot.co$\underline{\mathrm{m} / 20070401 \text { archive.html }}$

- Cuadro de comparación entre las teorías de Maslow, Herzberg y Alderfer, en

http://4.bp.blogspot.com/ qFEg7PeA PU/ SZeYQ-Z_ixI/AAAAAAAAADY/Hr7xXazsA80/s400/Imagen14.jpg

- Edutopia, Sage Advice: Challenging and Motivating Gifted Students, en

- http:// La pirámide de Maslow, en. http://adrian.otero.ws/imagenes/200709/piramide-maslow.jpg

- MÍGUEZ, Marina, extraído de http://revista.iered.org/vln $3 / \mathrm{html} / \mathrm{mmiguez} . \mathrm{html}$, disponible en http://www.emagister.com/motivacion-compresion-cursos-2438938.htm, 11 de Noviembre de 2006.

- www.edutopia.org/sage-advice-challenging-motivating-gifted-students

- Monereo Font, Carles, Estrategias de enseñanza y evaluación para mejorar la motivación de los estu- 
diantes, Depto. de Psicología básica, evolutiva y de la educación. Universidad autónoma de Barcelona, disponible en

- http://www.usal.es/gabinete/comunicacion/eEuropeo/Power Motivacion Universidad.pps

- REAl ACADEMIA ESPAÑOla, Diccionario de la Lengua Española, en http://buscon.rae.es/draeI

- Román Martiniano y Diez Eloisa: Currículum y aprendizaje. Madrid. 2005
- Tipos de motivación, en http://www.educared.edu.pe/aulashospitalariaslcompartiendo en red/assets c/2008/ 12/tipos\%20de\%20motivacion-thumb-500x685thumb-500x685.jpg

- Varios autores, La comunicación no verbal, blog disponible en

http://www.geocities.com/Athens/Column12150/page37.html

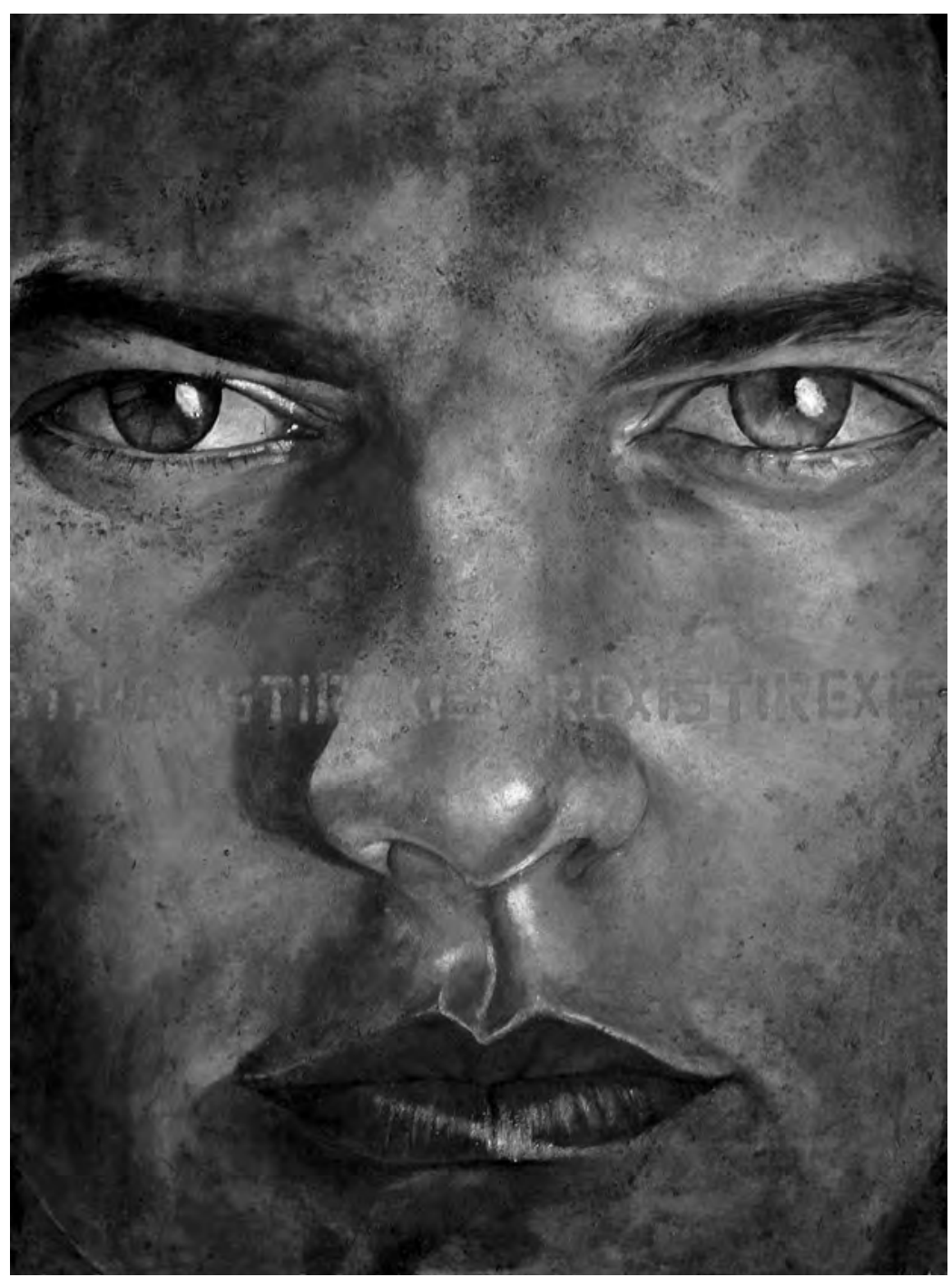

El otro. Acrílico sobre lona. 2008 


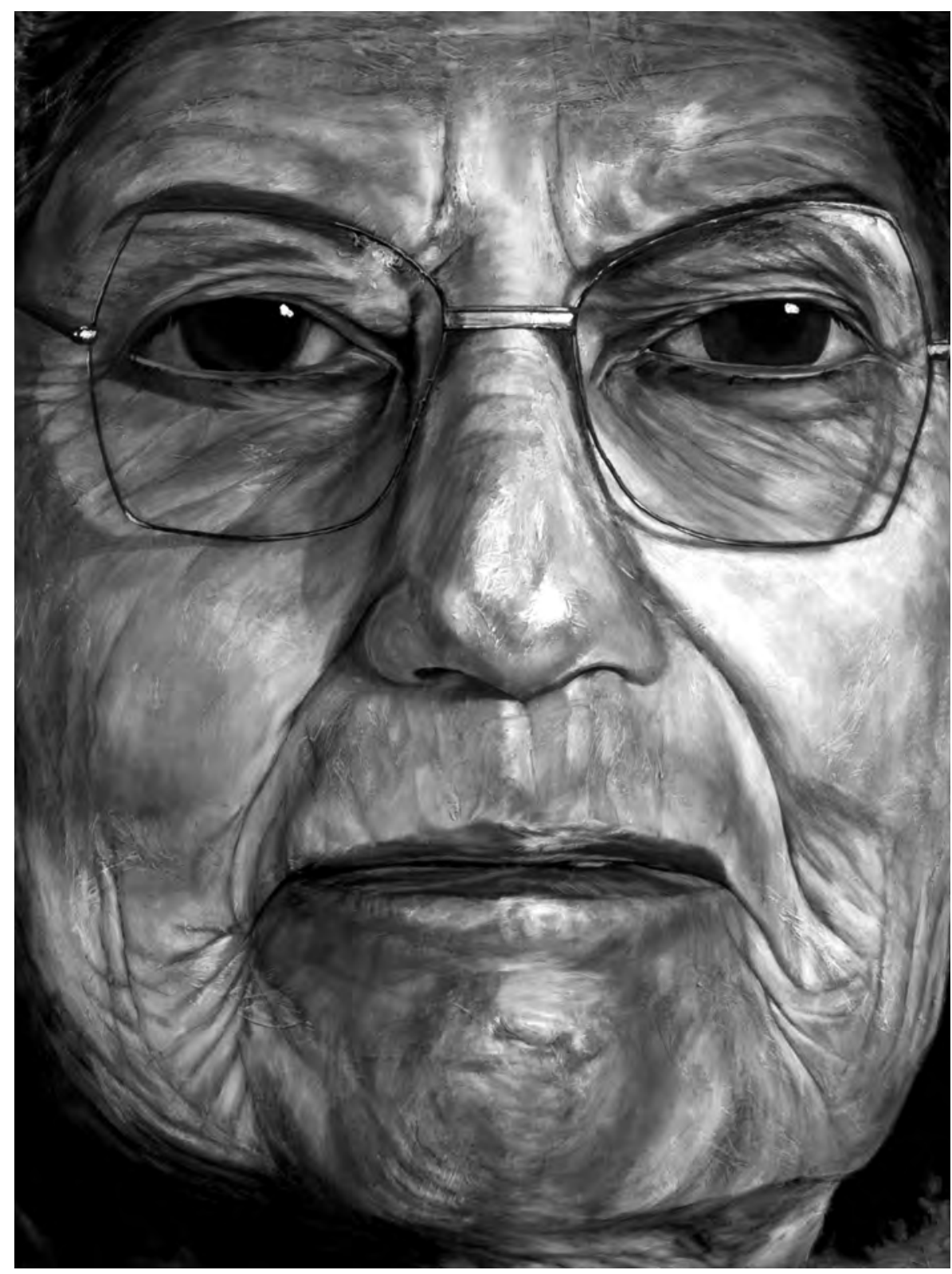

Quilago (32 módulos). Acrílico sobre tela. 2004 